\title{
On the linear stability of the inviscid Kármán vortex street
}

\author{
BY JAVIER JIMENEZ $\dagger$ \\ Applied Mathematics, California Institute of Technology, Pasadena, CA 91125, USA
}

(Received 3 March 1986)

The classical point-vortex model for a Kármán vortex street is linearly stable only for an isolated case. This property has been shown numerically to hold for other, more complicated, models of the same flow. It is shown here that it is a consequence of the Hamiltonian structure of the model, related to the codimension of the set of matrices with a particular Jordan block structure in the space of Hamiltonian matrices, and that it can be expected to hold generically for any two-dimensional inviscid array of vortices that has back-to-fore symmetry, and that is 'close enough' to the point-vortex model.

\section{Introduction}

The regular arrangement of vortices sometimes found in the wake of bluff bodies was first modelled by von Kármán (Lamb 1945) by a double infinite street of point vortices, moving with respect to the fluid at infinity at some constant velocity, and whose properties are characterized by a single parameter, $\eta=h / L$, which is the ratio between the separation of the two rows and the wavelength of the periodic vortex array. The stability of this arrangement to perturbations in the positions of the vortices was studied by von Kármán by expanding those perturbations in terms of normal modes, parameterized by a wavenumber $\kappa$. The stability of a given mode depends both on $\kappa$ and $\eta$, and a given street is stable when all the modes, $\kappa$, are stable for the given fixed value of $\eta$. It turns out that vortex streets are generally unstable, containing an unstable range of wavenumbers for all values of $\eta$ except for one,

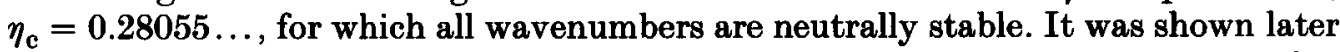
that, even in this case, the street exhibits a nonlinear instability (Domm 1956) due in essence to the fact that any small perturbation modifies $\eta$ enough to move the street into the unstable regime. Since natural wakes show regular vortex streets that persist for long times (see, for example, the beautiful pictures in Van Dyke 1982), it has usually been felt that a more complete model should produce a non-zero stability range of $\eta$ that would guarantee linear stability for streets contained inside that range.

In fact, for Reynolds numbers greater than approximately 100 , natural vortex streets undergo a process of slow disintegration and rearrangement (Taneda 1959), which is consistent with linear instability and which suggests that the stabilization mechanism might be viscous. Three-dimensionality appears at about the same time (Gerrard 1966), and two-dimensional models might not be applicable, but recent observations of two-dimensional wakes in thin films, as well as numerical evidence (Couder, Basdevant \& Thomé 1984), show a similar behaviour, although the transition Reynolds number appears to be higher (200), and the subsequent insta- 
bility mechanism is somewhat different. On the other hand, vortex streets form behind obstacles up to fairly large Reynolds numbers with experimental values of the spacing ratio $\eta$ which are close enough to the critical, $0.28 \ldots$ (see table I in Saffman \& Schatzman $1982 b$ ) to suggest some sort of relation with the fact that this is also the least unstable regime. This continues to be so even in cases for which the downstream evolution of the street is unstable.

It is therefore important to understand which is the stabilization mechanism responsible for the permanence of streets at low Reynolds numbers, and possibly for their formation at higher ones. Moreover, the existence of an isolated point of stability for the point-vortex model corresponds to a double root of some algebraic equation, which can be expected to disappear for neighbouring models, even if they are only small perturbations from the original one. The result would be either a non-zero stability range or no stability at all. This suggests that any perturbation to the point-vortex model should either be stabilizing or destabilizing near the critical spacing, and that it should be possible to obtain inviscid strongly stable vortex streets by perturbing the Kármán model.

A first attempt was done by Rosenhead (1929), who studied an infinite point-vortex model in a channel of finite width. Interestingly enough, for wide channels, the nature of the stability is unchanged, with an isolated, marginally stable value of $\eta$ that depends on the relative width of the channel. Recently, considerable attention has been given to arrays of regions of uniform vorticity and non-zero area, instead of point vortices (Christiansen \& Zabusky 1973; Kida 1982; Saffman \& Schatzman 1982a). After several unsuccessful attempts, it was shown by Meiron, Saffman \& Schatzman (1984) that the stability behaviour is again the same; at least for moderate values of the area of the individual vortices, there is a single value of $\eta$ for which the street is stable.

This persistent behaviour raises the questions of whether it is intrinsic to a wide class of inviscid flow models or whether it is specific to the models so far investigated, and, in the latter case, which features should be included in new models to produce the stability region that seems to be required by the experimental evidence.

We shall show below that the persistence of isolated points of stability is a generic feature of two-parameter families of conservative Hamiltonian systems which satisfy certain symmetries.

More precisely, consider a family of inviscid models for the Kármán street, having back-to-fore symmetry and depending continuously on any number of parameters, besides $\eta$ and $\kappa$, and which contains the classical point-vortex model as a special case. Any model in such a family will have a set of stability (collective) modes corresponding to the modes of the point-vortex model and which will evolve into them as the model approaches continuously the point-vortex case. In some finite neighbourhood of the point-vortex model, the stability exponents for the collective modes will depend continuously on $(\eta, \kappa)$, and we shall show that, for each particular value of all the extra parameters (e.g. vortex area or channel width) there is just an isolated value of $\eta$ for which the collective mode is stable. Of course, a model that is more complex than the point-vortex one may have other stability modes besides the collective one, such as internal oscillations of the finite-area vortices, and the arguments in this paper do not apply to them at all. They may be unstable, in which case the street will be unstable even for the critical value of $\eta$, but, even if they are always stable, the street as a whole will be unstable because of the collective instability. In any case, the present result precludes the existence of a non-zero range of stability around $\eta_{\mathrm{c}}$.

The arguments in this paper are local, in a sense to be made clear later, and may 
fail for models which are very different from the point-vortex case. Thus, they do not necessarily apply to vortex cores of large area, or to vortex streets in narrow channels, whose stability still has to be decided numerically. The computations in Meiron et al. (1984) were taken to fairly large values of the vortex area without any apparent broadening of the critical stability point, but the vortex street in a channel does develop a non-zero stability range when $h$ becomes a substantial fraction $(\approx 0.21)$ of the width of the channel.

In the following sections, the problem will be put first in mathematical terms, and related to known results for Hamiltonian systems. Then, the results will be applied to the vortex street and, finally, the appearance of a finite range of stability in the case of narrow channels will be treated and shown to be in accordance with the general theory. The results presented here make it unlikely that inviscid effects play an important part in stabilizing the vortex street. The effects found in natural flows are probably intrinsically dissipative.

\section{General formulation}

In problems dealing with linear stability of mechanical systems, the equations of motion can usually be written in terms of some perturbation vector $\hat{z}$, and an evolution matrix $A$, as

$$
\frac{\partial \hat{z}}{\partial t}=\boldsymbol{A} \hat{z}
$$

For the moment, think of $\hat{z}$ as having a finite number of components. As long as the eigenvalues of $\boldsymbol{A}$ remain simple, the temporal behaviour of $\hat{z}$ is given by a sum of terms proportional to $\mathrm{e}^{\sigma t}$, where $\sigma$ is an eigenvalue of $\boldsymbol{A}$. The sign of the real part of $\sigma$ determines the stability of the particular term involved, and the stability of the system is given by the properties of the spectrum of $\boldsymbol{A}$. We shall be specially interested in the case in which (1) derives from a Hamiltonian system. Then, the number of degrees of freedom is even, $2 N$, and Darboux's theorem (Arnol'd 1978) guarantees that there exist a canonical set of variables, $z=(p, q)$, such that, at least locally, the equations of motion can be written in the form

$$
\frac{\partial z}{\partial t}=\mathbf{I} \frac{\partial H}{\partial z}
$$

where $H$ is the Hamiltonian function, I is the canonical sympletic operator

$$
\left[\begin{array}{cc}
0 & -\boldsymbol{U} \\
\boldsymbol{U} & 0
\end{array}\right]
$$

and $\boldsymbol{U}$ is the $N \times N$ unit matrix.

If we refer now to an equilibrium point of (2) such that $\partial H / \partial z=0$, and let the system perform small motions around it, the linear evolution equations for the perturbation quantities, $\hat{z}=(\hat{p}, \hat{q})$, would have the form

$$
\frac{\partial \hat{z}}{\partial t}=\mathbf{I S} \hat{z}
$$

where $S=\partial^{2} H / \partial z^{2}$, is the symmetric Hessian of the Hamiltonian function. Matrices of the form $I S$, in which $S$ is real and symmetric, are usually called Hamiltonian, and some of their properties are summarized in Appendix 6 of Arnol'd (1978).

Their first useful property is that, if $\sigma$ is an eigenvalue of IS, so is $-\sigma$ and, since the matrix is real, so are their complex conjugates $\bar{\sigma}$ and $-\bar{\sigma}$. Therefore, generic 
complex eigenvalues come in quartets, which coalesce into pairs in the particular cases in which $\sigma$ is either real or pure imaginary. Moreover, the multiplicity and the Jordan block structure of all the members of a quartet is the same. We shall be interested here in the codimension, in the space of matrices of a given form, of the sets of matrices with a given Jordan block structure.

The codimension of a manifold in a general space is the number of dimensions left to fill the space. Sets of codimension 1 are equivalent to surfaces in 3-space, and they divide the space into disjoint regions. Sets of codimensions 2 and 3 correspond to isolated lines and points, and higher codimensions define, in higher-dimensional space, sets which are even sparser. The advantage of using codimensions is that, while the dimension of a manifold may depend on the dimension of the space in which it is imbedded, the codimension tends to be independent of that, and to be a function only of the properties defining the manifold. Also, if we consider an $m$-parameter family of points defined in a given space, it will generically intersect a manifold of codimension $c$ at a set of dimension $m-c$. In particular, those families for which $m<c$, will not intersect the manifold at all. These are generic properties, to be understood in the same sense that a line will always intersect a surface in 3-space at a point, while two lines will never intersect.

The structure of the family of Hamiltonian matrices with respect to their Jordan block structure is given by a theorem due to Galin (1975) (see Arnol'd 1978, Appendix 6). The generic (codimension 0) state for a Hamiltonian matrix is to have all its eigenvalues different. Note that, in each pair of real eigenvalues or in each complex quartet, there is always one eigenvalue with positive real part, which makes the evolution equation (4) unstable. The only case in which the system is stable is when all the eigenvalues belong to pure imaginary pairs. In a continuous, one-parameter family of Hamiltonians, the transition from stability to instability usually occurs when two imaginary pairs collide and fly off the imaginary axis to form a complex quartet. At the point of collision, the Hamiltonian perturbation matrix has a pair of double pure imaginary eigenvalues. The only other fundamentally different mechanism for stability exchange occurs when several eigenvalues vanish simultaneously, as is the case for the vortex street in the limit of very long wavenumbers.

The generic Jordan structure for a Hamiltonian matrix with a double pair of eigenvalues is to have a pair of non-trivial $2 \times 2$ blocks. The set of matrices with this structure had codimension 1 in the space of all Hamiltonian matrices and, therefore, represents a set of surfaces that divide the space into regions, each of which may have different stability. The matrices which fall in the intersections of these surfaces also have a pair of double eigenvalues, but these eigenvalues each generate a pair of $1 \times 1$ Jordan blocks. The dimension of the largest non-trivial Jordan block associated to an eigenvalue is called its index. From Galin's theorem, the set of matrices having a pair of double eigenvalues of index 1 has codimension 3 in the space of Hamiltonian matrices and, as a consequence of the analytic behaviour of the eigenvalues in the neighbourhood of such matrices, it generally lies in the vertex of cones formed by the surfaces which contain the double eigenvalues of index 2 (figure 1a), although other behaviours are possible (MacKay 1986).

It will be shown below that, when we only consider matrices satisfying certain symmetry conditions, the set of index-1 double eigenvalues has codimension 2 . In this case it would generally lie in the edges formed at the intersections of the index-2 surfaces and the geometry would be equivalent to the one in figure $1(b)$.

The significance of these properties for the stability of the Kairmán vortex street is this. The perturbation modes of a given vortex street correspond to a continuous 


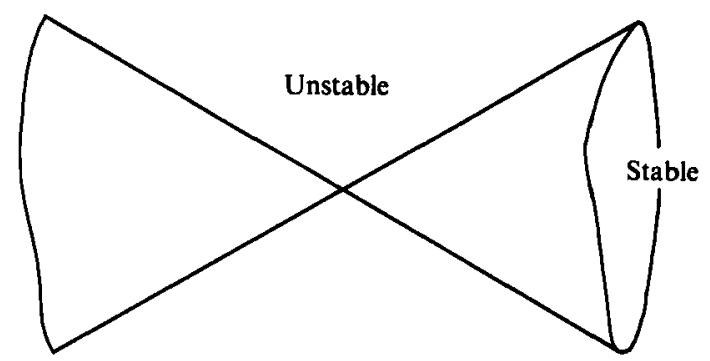

(a)

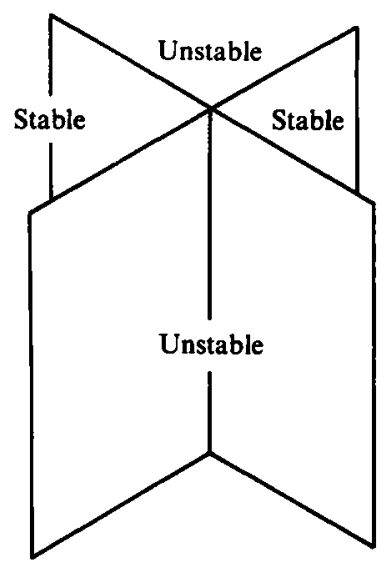

Figure 1. Structure of the set of matrices with double eigenvalues in the neighbourhood of the subset that has double eigenvalues with index 1. (a) General Hamiltonian matrices. (b) Case in which the subset has codimension 2 .

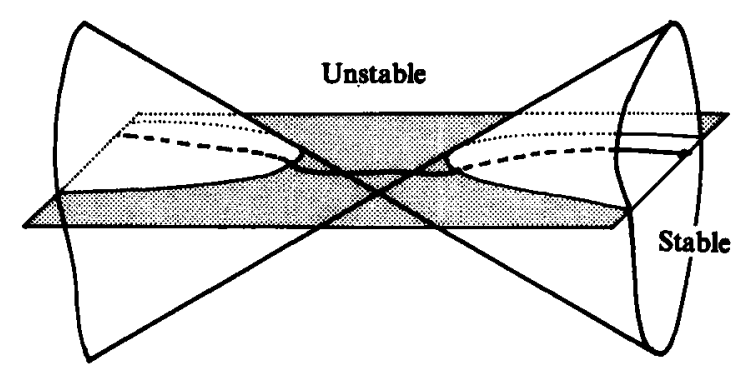

Figure 2. All the one-parameter families in a two-parameter family of matrices will generically intersect the unstable region in the space of general Hamiltonian matrices.

one-parameter $(\kappa)$ family of evolution matrices. This family forms a line in the space of matrices, and can be expected to intersect the codimension-1 set of matrices with generic double eigenvalues, and to have some modes that fall in the stable, and some in the unstable, region of the space of matrices (figure 2). If we now consider a one-parameter $(\eta)$ family of vortex streets, each of them corresponding to a one-parameter $(\kappa)$ family of perturbation modes, we get a two-parameter $(\eta, \kappa)$ manifold of stability matrices. In the case of general Hamiltonian matrices (figure 2) this surface will not generally intersect the vertex of the cone, and all the one-parameter lines that represent individual streets can be expected to lie part inside and part outside the stable region. Any such street contains some unstable modes and is, therefore, unstable.

This is the reason why it looks a priori that the possibility of finding a vortex street which is barely stable (crossing through the vertex), in a one-parameter family of streets, is structurally unstable and should break down one way or another under small perturbations. If, however, it can be shown that the evolution of the modes in a vortex street has enough symmetries that the index-1 manifold has codimension 2 , it is clear that any two-parameter family would generically intersect the edge of index-1 matrices, and that any such family would generally include a one-parameter line crossing the edge and barely avoiding the unstable region (figure 3 ). In this case, 


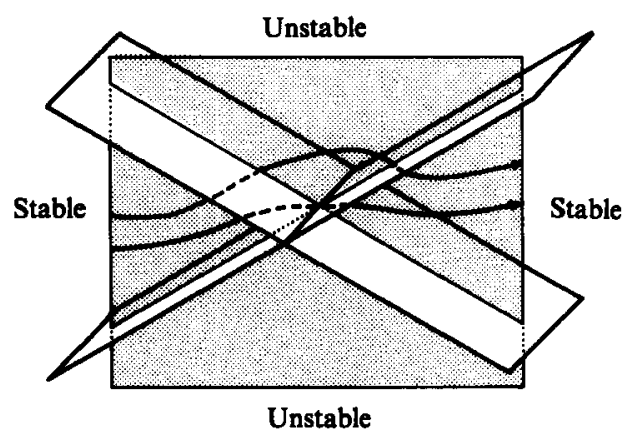

FraUre 3. Any two-parameter family of matrices will generically contain one isolated line that avoids the unstable region in a space of matrices in which the index-1 subset has codimension 2 .

therefore, any one-parameter family of vortex streets can be expected to contain at least one barely stable case which would correspond to an isolated member of the family.

\section{The vortex street}

A two-dimensional set of point vortices, each with a circulation $\gamma_{j}$, has long been known to be a Hamiltonian system (Batchelor 1967). The Hamiltonian function is given by

$$
H=\frac{1}{2} \sum_{j} \sum_{k} \gamma_{j} \gamma_{k} \log \left[\left(x_{j}-x_{k}\right)^{2}+\left(y_{j}-y_{k}\right)^{2}\right] \text {, }
$$

and a canonical set of variables is $(p, q)=\left\{x_{j}, \gamma_{j} y_{j}\right\}$. Since any two-dimensional vorticity distribution can be thought of as a superposition of an infinite number of point vortices, it is at least plausible that a similar Hamiltonian structure exists for more complicated distributions. Indeed, J. Gibbons (1985, private communication) has given a Hamiltonian formulation for sets of smooth patches of uniform vorticity. His Hamiltonian is a straight generalization of (5), and the structure is written in terms of the normal displacements of the boundary of the patches. He also gives canonical variables for the particular case of almost circular vortices. Note, however, that even if canonical variables are not explicitly known, their existence is guaranteed by Darboux's theorem, and that this existence is enough for the arguments in this paper.

More complicated, non-uniform, patches can be built by superposition of concentric bands with different vorticities, and the Hamiltonian of these patches would just be the sum of the Hamiltonians of the different uniform bands. In the limit of an infinite number of bands, it should be possible to construct Hamiltonians for arbitrary continuous vorticity distributions, but the details of that limit have to be done with care, and that case is not considered here. In fact Arnol'd (1969) has shown that the Euler equations, for incompressible, inviscid flow with an arbitrary vorticity distribution have a Hamiltonian formulation as long as permissible motions are restricted to volume-preserving deformations that introduce no new vorticity. A review can be found in Marsden \& Weinstein (1983), which also treats the special case of uniform vortex patches. The discussions in this paper should apply to the general case, but the limiting process is complicated owing to the possibility of the appearance of a continuous spectrum for the stability equations.

Even for a single vortex patch the number of degrees of freedom is infinite, and the application of the discussion in the previous section has to be done carefully. In 
general, those results should hold as long as the infinite-dimensional system can be considered as the limit of a sequence of finite-dimensional ones, and as long as we are interested in the properties of a particular eigenvalue which can be defined as the proper limit of a given sequence of eigenvalues of the finite-dimensional problems, and which remain discrete during the limiting process. When the eigenvalues form a continuous range, the concept of a Jordan block itself has to be redefined.

The characteristic frequencies for a circular uniform vortex patch were studied first by Kelvin (see Lamb 1945, $§ 158$ ), and shown to be discrete and well separated. Gibbon's canonical variables are given in terms of the Fourier expansion for the shape of the contour, and the limit from a finite number of degrees of freedom is well defined, at least for almost circular patches, and as long as we are interested in the behaviour of a particular finite-order harmonic. By continuity, its eigenvalues are also separated. The same is true for any equilibrium arrangement of a finite number of vortex patches, again as long as they stay far enough from each other to remain nearly circular, but, for the infinite periodic arrangement in the Kármán street, the isolated eigenvalues broaden into continuous segments, for which the previous discussion does not hold, and some transformation has to be introduced to make the spectrum once more discrete.

The modal expansion used by Kármán reduces the system again to one with discrete eigenvalues, but at the price of introducing a new continuous parameter $\kappa$, which essentially indexes the continuous eigenvalue segments. Consider a periodic arrangement of identical vortex systems, each of them defined by canonical perturbation coordinates $\hat{z}_{n}, n=-\infty, \ldots, \infty$. The equations of motion have the form

$$
\frac{\partial \hat{z}_{n}}{\partial t}=\mathbf{I S}_{n m} \hat{z}_{m}
$$

where the matrices $S_{n m}=\partial^{2} H / \partial z_{n} \partial z_{m}$ are real and symmetric in the sense that $\boldsymbol{S}_{n m}^{\prime}=\boldsymbol{S}_{m n}$, and where the prime stands for matrix transposition. Moreover, since the system is invariant under translation by one period, $S_{n m}$ is only a function of $n-m$ and, under those conditions, it is easy to see that the eigenvectors of (6) have the form $\hat{z}_{n}=\tilde{z} \mathrm{e}^{\mathrm{i} \kappa \boldsymbol{n}}$, and satisfy

$$
\frac{\partial \bar{z}}{\partial t}=\mathbf{I} \overline{\boldsymbol{z}}, \quad T=\sum_{-\infty}^{\infty} \boldsymbol{S}_{n m} \mathrm{e}^{\mathrm{i} \kappa(m-n)} .
$$

The stability of (7) is again controlled by the spectrum of $\boldsymbol{T}$.

The symmetry of the $\boldsymbol{S}_{n m}$ implies that $\boldsymbol{T}$ is Hermitian, which is not enough to make IT Hamiltonian. However, when the system is symmetric in the $n \rightarrow-n$ direction, as in the case of the vortex street, it is possible to write $T$ as a pure imaginary matrix, and thus recover some of the properties of Hamiltonian systems.

Consider Gibbon's Hamiltonian formulation in more detail. The form given here is a slightly generalized one in which the vorticity of the patches is explicitly included, which is necessary in studying systems, like the vortex street, in which different patches have different vorticities. Assume that we have a set of patches, for each of which the vorticity $\omega$ is constant. Denote as $\Omega$ the domain, formed by several subdomains, occupied by the vortices in the plane $r=\{x, y\}$, and let its contour be parameterized by an arbitrary parameter as $\boldsymbol{r}(s)$ (which would contain one circuit per vortex patch). The Hamiltonian is then given by

$$
H=\int_{\Omega} \int_{\Omega} \omega\left(r_{1}\right) \omega\left(r_{2}\right) \log \left|r_{1}-r_{2}\right| \mathrm{d}^{2} r_{1} \mathrm{~d}^{2} r_{2}
$$


The dynamical variable is the oriented area displacement of the contour, defined by

$$
\delta A(s)=-\frac{\partial r}{\partial s} \wedge \delta r(s)
$$

and the equations of motions are

$$
\frac{\partial A(s)}{\partial t}=\omega^{-1}(s) \frac{\partial}{\partial s} \frac{\partial H}{\partial A}(s) .
$$

The canonical variables are those in which the operator $\omega^{-1}(s) \partial / \partial s$ takes the form (3). They can be constructed in two steps: consider first a set of variables $\{\boldsymbol{p}(s), \boldsymbol{q}(s)\}$ that reduces $\partial / \partial s$ to canonical form; a canonical set for the complete operator is then $(\boldsymbol{p}, \boldsymbol{Q})=\{\boldsymbol{p}(s), \omega(s) \boldsymbol{q}(s)\}$.

Consider now the behaviour of these variables and of the matrices in (7) under the symmetry $n \rightarrow-n$. The variables $p$ and $q$ are just oriented areas, and they change sign on reflection. So does the vorticity $\omega(s)$, and $\mathrm{e}^{\mathrm{i} \kappa n}$ goes into its complex conjugate $\mathrm{e}^{-\mathrm{i} \kappa n}$. The Hamiltonian itself remains unchanged. Therefore $(p, Q) \rightarrow(-p, Q)$, and the matrices $S_{n}$, which have the form

$$
\left[\begin{array}{cc}
\frac{\partial^{2} H}{\partial p^{2}} & \frac{\partial^{2} H}{\partial p \partial Q} \\
\frac{\partial^{2} H}{\partial Q \partial p} & \frac{\partial^{2} H}{\partial Q^{2}}
\end{array}\right] \rightarrow\left[\begin{array}{ll}
\frac{\partial^{2} H}{\partial p^{2}} & \frac{-\partial^{2} H}{\partial p \partial Q} \\
\frac{-\partial^{2} H}{\partial Q \partial p} & \frac{\partial^{2} H}{\partial Q^{2}}
\end{array}\right]
$$

As a consequence, the matrix $T$ transforms as

$$
\left[\begin{array}{ll}
T_{11} & T_{12} \\
T_{21} & T_{22}
\end{array}\right] \rightarrow\left[\begin{array}{rr}
T_{11} & -T_{12} \\
-T_{21} & T_{22}
\end{array}\right],
$$

where the bar denotes complex conjugation. Since (7) should be left invariant by the reflection, both sides of (12) are equal, and the structure of $T$ is

$$
\boldsymbol{T}=\left[\begin{array}{rr}
\boldsymbol{R}_{11} & \mathrm{i} \boldsymbol{R}_{12} \\
-\mathrm{i} \boldsymbol{R}_{12}^{\prime} & \boldsymbol{R}_{22}
\end{array}\right],
$$

where all the $\boldsymbol{R}$ 's are real, and $\boldsymbol{R}_{11}$ and $\boldsymbol{R}_{22}$ are symmetric. Taking now as variables $(\tilde{\boldsymbol{p}}, \mathrm{i} \boldsymbol{Q}),(7)$ becomes

$$
\frac{\partial \tilde{z}}{\partial t}=\mathrm{i} \boldsymbol{R} \tilde{z}, \quad \boldsymbol{R}=\left[\begin{array}{rr}
\boldsymbol{R}_{11} & \boldsymbol{R}_{12} \\
-\boldsymbol{R}_{12}^{\prime} & -\boldsymbol{R}_{22}
\end{array}\right],
$$

where $\boldsymbol{R}$ is real but, unfortunately, not symmetric, which would be necessary for a Hamiltonian structure. The problem is that, even for spatially symmetric systems, the forward and backwards directions along the vortex street are not exactly equivalent, owing to the sense of rotation imposed by the vorticity. As a consequence, the Hamiltonian structure of the original system becomes distributed between the $\kappa$ and the $-\kappa$ stability modes, neither of which stays exactly Hamiltonian.

Even so, some Hamiltonian properties hold for each of the two new systems. Consider the equations of motion of the modal variables associated with $-\kappa$. From (7), the matrix associated to this mode is just the complex conjugate of $T$. Also, because (7) can be written in the purely imaginary form (14), its eigenvalues come 
in pairs $(\sigma, \bar{\sigma})$, while those associated with $-\kappa$ contain the missing part of the quartet, $(-\sigma, \bar{\sigma})$. Consider now the composite system

$$
\frac{\partial}{\partial t}\left[\begin{array}{c}
\tilde{z}(\kappa) \\
\tilde{z}(-\kappa)
\end{array}\right]=\left[\begin{array}{cc}
\mathbf{I T} & 0 \\
0 & \mathbf{I T}
\end{array}\right]\left[\begin{array}{c}
\tilde{z}(\kappa) \\
\tilde{z}(-\kappa)
\end{array}\right] .
$$

Since the matrix is block-diagonal, its eigenspace is the direct sum of the eigenspaces of the two blocks, and its eigenvalues are just the eigenvalues of the two blocks with their respective multiplicities and Jordan block structure. Moreover the composite system now has the eigenvalue quartets characteristic of Hamiltonian matrices and, in fact, it is easy to see by direct substitution that, when (15) is written in terms of the variables $\boldsymbol{Z}=\{\tilde{\boldsymbol{p}}(\kappa), \tilde{\boldsymbol{p}}(-\kappa), \mathrm{i} \boldsymbol{Q}(-\kappa), \mathrm{i} \boldsymbol{Q}(\kappa)\}$, it takes the form

$$
\frac{\partial \boldsymbol{Z}}{\partial t}=\mathrm{il} \boldsymbol{F} \boldsymbol{Z}, \quad \boldsymbol{F}=\left[\begin{array}{cccc}
0 & \boldsymbol{R}_{11} & -\boldsymbol{R}_{12} & 0 \\
\boldsymbol{R}_{11} & 0 & 0 & \boldsymbol{R}_{12} \\
-\boldsymbol{R}_{12}^{\prime} & 0 & 0 & -\boldsymbol{R}_{22} \\
0 & \boldsymbol{R}_{12}^{\prime} & -\boldsymbol{R}_{22} & 0
\end{array}\right]
$$

Since $\boldsymbol{F}$ is real and symmetric, the matrix $\boldsymbol{F}$ is Hamiltonian, which was to be expected since (16) contains the full behaviour of the original Hamiltonian system.

Consider now the collapse of a complex eigenvalue quartet of the block-diagonal matrix in (15) into a double pair of pure imaginary eigenvalues. First, there is a one-to-one correspondence between matrices of the form (15) and either $\boldsymbol{T}$ or $\boldsymbol{T}$ and, in fact, any one of these three matrices defines the other two completely. Therefore, the three matrix spaces are isomorphic. Then, all the eigenvalues of the composite system belong either to the $\kappa$ or the $-\kappa$ modes and, from the discussion above on the eigenvalue pairs associated with each mode, the collapse happens in such a way that the double pure imaginary eigenvalue $\sigma$ corresponds to one mode, while the double eigenvalue $-\sigma$ corresponds to the other. As a consequence, the sub-manifold of matrices with a double pure imaginary eigenvalue pair (of a given index) in the space of composite matrices (15) is identical with the sub-manifold of matrices with a double pure imaginary eigenvalue in the space of either $\boldsymbol{T}$ or $\boldsymbol{T}$, and the codimensions of each of the three (isomorphic) manifolds in their corresponding spaces are the same. It turns out that the particular Hamiltonian form in (16) has enough symmetries that the codimension of double index-1 real eigenvalue pairs of $\boldsymbol{F}$ is equal to 2 (see the Appendix) and, therefore, the same property holds for pure imaginary eigenvalue pairs of (7). Note that these conclusions would not apply, for example, to double real eigenvalues of (7) since, in that case, it is possible to have a double eigenvalue of (15) in which one member of the pair belongs to $T$, and the other to $T$, and the corresponding manifolds are not necessarily isomorphic. Fortunately, those pairs are not important for stability.

The eigenvalues associated with the collective mode of (7) are isolated for the point-vortex model of the Kármain street, and remain so for small perturbations around it. Therefore, the conclusions in the previous discussion apply, and the property that any one-parameter (e.g. $\eta$ ) family of vortex streets contains an isolated member with marginal instability, surrounded by unstable members, can be expected to remain true under small perturbations to the model. This holds for perturbed point models, such as the flow in a channel, for small uniform vortex patches, and even for non-uniform vortices, at least for those built from a finite number of concentric 
uniform bands. It is probably also true for more general non-uniform vortices, but this is subject to clarification of the limit process mentioned earlier. This conclusion agrees with the numerical evidence.

\section{Transversality}

That two manifolds have the right codimensions does not guarantee that they actually cross, in the same way that two lines do not necessarily intersect in the plane. The useful result is that, if two manifolds do cross and have the right dimensionality, they will continue crossing after a small perturbation of the problem. Even this is not always true, as in the case of two tangent curves that separate after a small deformation.

The key concept is that of transversality (Shirer \& Wells 1983). Two linear subspaces are called transversal in a given space if, between them, they contain enough vectors to span the full space. Two manifolds cross transversely at a point if their tangent spaces at that point are transversal and, in that case, it is true that the intersection, and transversality itself, are stable to small perturbations. It is easy to see that the codimension condition given above, that the sum of the dimensionalities of both manifolds is at least equal to the dimensionality of the full space, is necessary for transversality. This condition is not sufficient, and counterexamples are all those manifolds that touch tangentially, and whose intersections are not necessarily stable to small perturbations. The intuitive notion of transversality implies that the two manifolds cross at an angle.

Since transversal intersections are structurally stable, it is enough to prove transversality for the intersection of two manifolds in some particular reference case to ensure that they will continue crossing each other after being perturbed slightly about that reference position.

The two-parameter family of modes for the point model of the vortex street cuts transversely the family of matrices with double eigenvalues of index 1 . This is the reason why its stability properties are maintained for all models which can be considered as small perturbations around it. In fact, the intersection of the two manifolds in the case of the point-vortex model is strongly transversal (actually, orthogonal) and situated far from any border in both manifolds, and, to loose it, either the angle or the point of intersection would have to be modified by an amount which is $O(1)$. If the parameters of any modified model are scaled in such a way that all functional derivatives are also $O(1)$, the intersection, and the stability properties, will be maintained in a region of that same order of magnitude.

Transversality is easy to prove for the point-vortex model. In that case, the two $( \pm \kappa)$ modes are identical, and each of them breaks into two symmetry modes, whose stability properties are equivalent. In each case, the evolution matrix is $2 \times 2$, and has the form

$$
\frac{\partial \tilde{z}}{\partial t}=\mathrm{i} \boldsymbol{M} \tilde{z}, \quad \boldsymbol{M}=\left[\begin{array}{ll}
b & a \\
c & b
\end{array}\right]
$$

where the two equal diagonal elements are a consequence of the Hamiltonian nature of the original system. Clearly, those matrices form a three-dimensional space, in which matrices with double eigenvalues of index 2 lie on the two planes $a=0$ and $c=0$, while those with a double eigenvalue of index 1 lie on the intersection of these two planes on the $b$-axis. A tangent vector to this last manifold is

$$
M_{1}=\left[\begin{array}{ll}
1 & 0 \\
0 & 1
\end{array}\right]
$$


Choosing one of the two equivalent symmetry modes, the family of matrices that represent the vortex street is (Lamb 1945)

$$
\left.\begin{array}{rl}
a & =C+A, \quad c=C-A, \\
b & =\frac{\pi \kappa \sinh \eta(\pi-\kappa)}{\cosh \pi \eta}+\frac{\pi^{2} \sinh \kappa \eta}{\cosh ^{2} \pi \eta}, \\
A & =\frac{1}{2} \kappa(2 \pi-\kappa)-\frac{\pi^{2}}{\cosh ^{2} \pi \eta}, \\
C & =\frac{\pi^{2} \cosh \kappa \eta}{\cosh ^{2} \pi \eta}-\frac{\pi \kappa \cosh \eta(\pi-\kappa)}{\cosh \pi \eta}
\end{array}\right\}
$$

The other symmetry mode simply changes the sign of $b$. At each point, the tangent space to this family is spanned by $\partial M / \partial \kappa$ and $\partial M / \partial \eta$. At the point of intersection of (19) with the index-1 subspace, $\kappa=\pi, \cosh ^{2} \pi \eta=2$, and

$$
\frac{\partial M}{\partial \kappa} \sim\left[\begin{array}{ll}
0 & 1 \\
1 & 0
\end{array}\right], \quad \frac{\partial M}{\partial \eta} \sim\left[\begin{array}{rr}
0 & 1 \\
-1 & 0
\end{array}\right] .
$$

It is clear that matrices (18) and (20) span the full space of matrices of the form (17) and, therefore, that the intersection of the two manifolds is transversal.

It has been pointed out by R. S. MacKay in a paper in preparation that, because the eigenvalues of the $\kappa$-mode are identical with those of $-\kappa$ in the point-vortex model, all the eigenvalues of (15) are double in that case, and they collapse to a pair of quadruple eigenvalues at the critical point. The results in the previous section would not apply to that case. This is only superficially so. Under small perturbations, the two eigenvalue quartets, $\{\sigma,-\sigma, \bar{\sigma},-\bar{\sigma}\}$, which are identical in the point-vortex case, will change into two new quartets, no longer necessarily identical. The arguments in the Appendix show that the collapse of each of these quartets into double pairs is a codimension-2 event, and the results of the previous section show that each one of the resulting double eigenvalues belongs to either $\kappa$ or $-\kappa$. Both arguments are independent of whether the quartets are simple or double, as long as there is no degeneracy between them, which is shown by the fact that (15) is block diagonal. As a consequence, any two-parameter family of matrices of the form (15), will contain two isolated stable members, each one corresponding to the collapse of one of the two eigenvalue quartets. It is not immediate that those two members correspond to the same street (same value of $\eta$ ), but that is consequence of the symmetry between the $\kappa$ and $-\kappa$ (or, equivalently, $2 \pi-\kappa$ ) modes. It follows from (15) that, if the mode $(\eta, \kappa)$ has a double eigenvalue $\mathrm{i} \sigma$ with a given Jordan structure, the mode $(\eta, 2 \pi-\kappa)$ has an eigenvalue $-\mathrm{i} \sigma$ with an equivalent structure. Therefore, the stability map for the two-parameter family takes the form in figure 4, and there is still a single value of $\eta$ for which the street is stable.

We consider now the vortex street in a channel, which eventually develops a non-zero stability interval, and seems to contradict these conclusions. Actually, the Rosenhead (1929) stability analysis is wrong in that he uses $\cos \kappa n$ expansions for his perturbation modes instead of $\mathrm{e}^{\mathrm{i} \kappa n}$. This is equivalent to forcing $b=0$ in (17), and results in standing, instead of travelling, perturbation waves. Aside from that, the stability characteristics do not change, and the system is a useful one in which to study the effects of loss of transversality. 


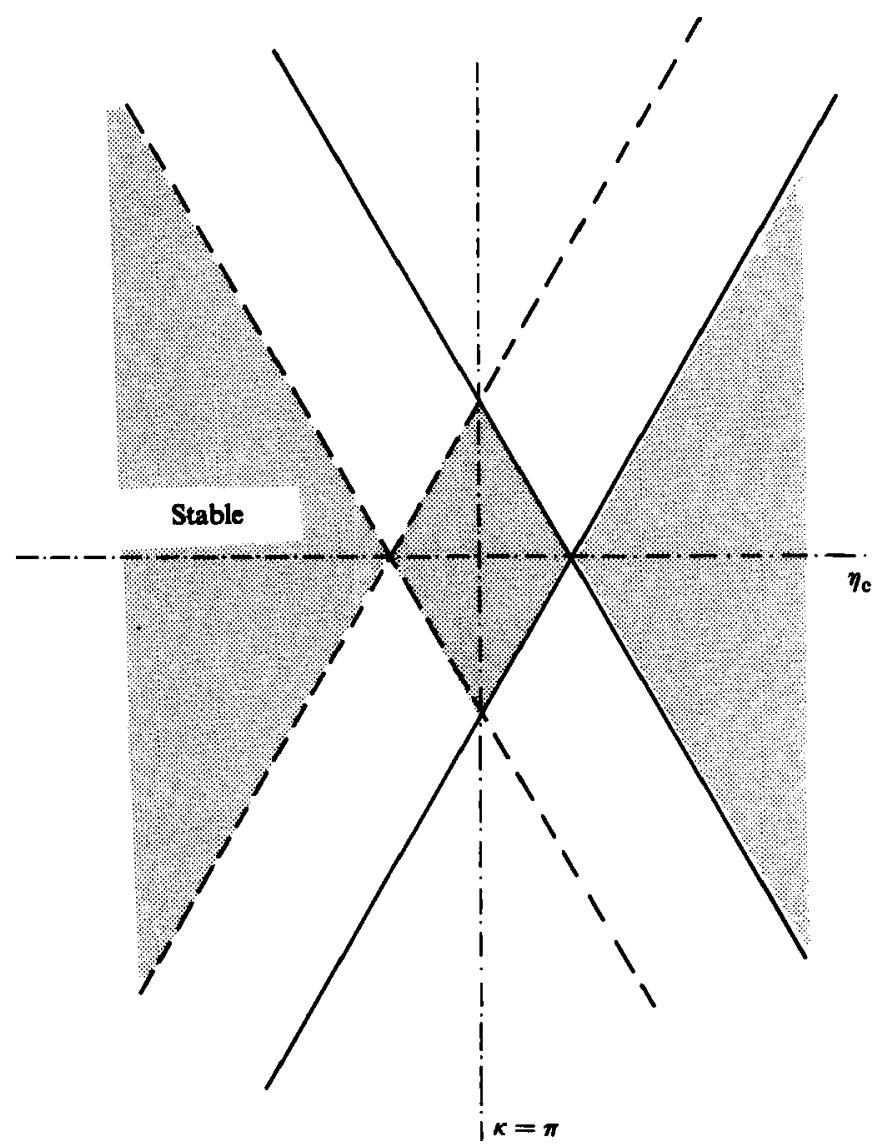

Fiqure 4. Stability diagram for a generic one-parameter family of vortex streets satisfying the conditions in the text. For the particular case of the point-vortex model, the two crosses collapse into a single one at $\kappa=\pi$, and the stability diamond in the centre of the diagram disappears.

The general structure of the matrix is the same as in the Kármán model, with different expressions for $a$ and $c$,

$$
\begin{aligned}
a= & -\sec ^{2} \nu / 2-2 \sum_{n=1}^{\infty} \operatorname{sech} n \mu\left[(1+\cos n \kappa) \frac{\cos \nu+\operatorname{sech} n \mu}{(1+\cos \nu \operatorname{sech} n \mu)^{2}}+\frac{1-\cos n \kappa}{1-\operatorname{sech} n \mu}\right] \\
& +2 \sum_{n=1}^{\infty} \operatorname{sech} n^{\prime} \mu\left[\left(1+\cos n^{\prime} \kappa\right) \frac{\cos \nu-\operatorname{sech} n^{\prime} \mu}{\left(1-\cos \nu \operatorname{sech} n^{\prime} \mu\right)^{2}}+\frac{1-\cos n^{\prime} \kappa}{1+\operatorname{sech} n^{\prime} \mu}\right], \\
c= & -2 \sum_{n=1}^{\infty} \operatorname{sech} n \mu(1-\cos n \kappa)\left[\frac{\cos \nu+\operatorname{sech} n \mu}{(1+\cos \nu \operatorname{sech} n \mu)^{2}}+\frac{1}{1-\operatorname{sech} n \mu}\right] \\
& +2 \sum_{n=1}^{\infty} \operatorname{sech} n^{\prime} \mu\left(1-\cos n^{\prime} \kappa\right)\left[\frac{\cos \nu-\operatorname{sech} n^{\prime} \mu}{\left(1-\cos \nu \operatorname{sech} n^{\prime} \mu\right)^{2}}+\frac{1}{1+\operatorname{sech} n^{\prime} \mu}\right], \\
n^{\prime}= & n-\frac{1}{2},
\end{aligned}
$$

and $b=0$. In this family there are two geometric parameters $\mu=\pi L / W$ and $\nu=\pi h / W$. The meaning of $h, L$ and $\kappa$ is the same as before, and $W$ is the width of the channel. The matrices now form a two-dimensional space $(a, c)$, in which the double eigenvalue, index-1, manifold is just the origin of coordinates. 


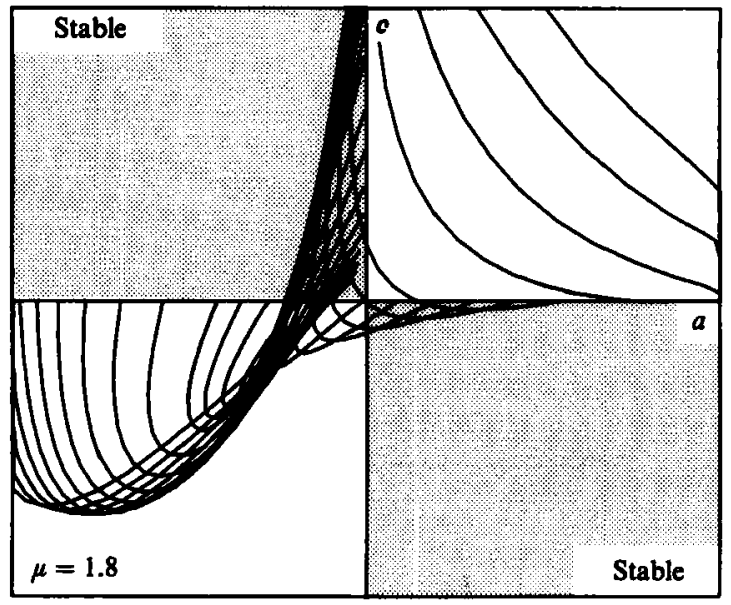

(a)

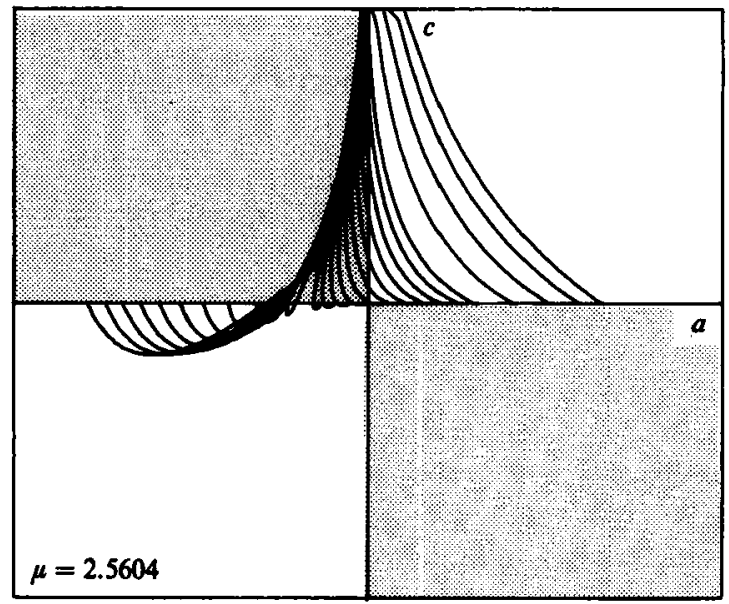

(b)

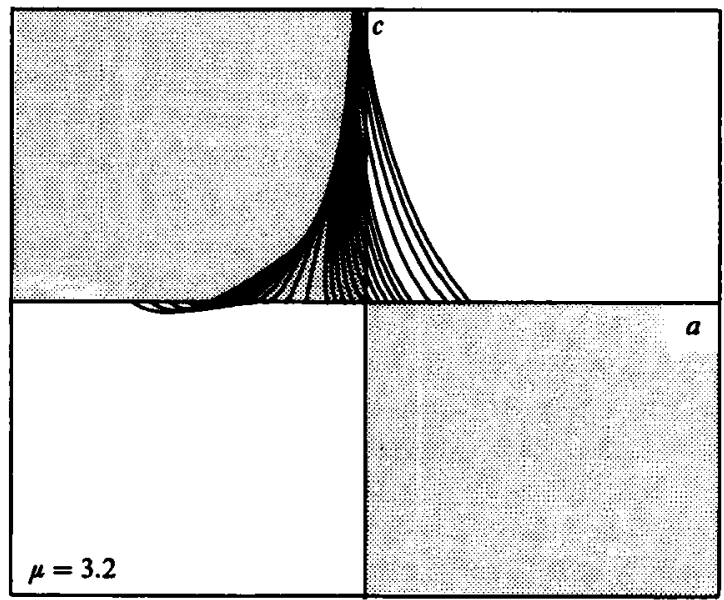

(c)

Figure 5. The two-parameter families of matrices representing the point-vortex street in a channel that is wider $(a)$, equal $(b)$, or narrower $(c)$ than the critical width necessary for the appearance of a stable region. Individual lines are complete streets with $\nu$ constant and a full range of $\kappa$. The range of $v$ 's represented in each case can be read from figure 6 . 


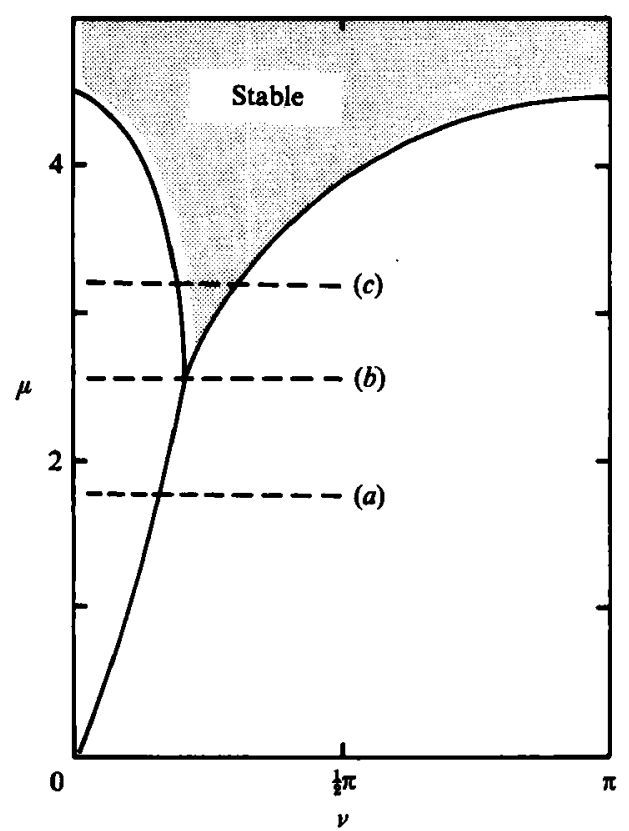

FIGURE 6. Stabiity diagram for the point-vortex street in a channel. Dashed lines represent the families plotted in figure 5 .

The expressions in (21) are complicated and, to study transversality, we would need to compute the derivatives with respect to the various parameters. Without doing that explicitly, it is easy to see that both $a$ and $c$ are periodic in $\kappa$ with period $4 \pi$, and it can be shown that $\kappa=0$ and $\kappa=2 \pi$ are turning points of their graphs with respect to $\kappa$. What really happens is that these graphs are not infinite lines, but finite segments, and that $\kappa=0$ and $2 \pi$ correspond to the end points of those segments.

Consider now a two-parameter family of modes (say $\nu, \kappa)$. The individual vortex streets would be represented by segments in the $(a, c)$-plane, and all the segments corresponding to different $\nu$ 's would fill a region. In this plane the two coordinate axes are stability boundaries, with the first and third quadrants containing unstable modes, and the second and fourth, stable ones. Normally, each segment, representing an individual vortex street, would cross into several quadrants and contain both stable and unstable modes. One of the segments in the region would cut the origin and correspond to an isolated, marginally stable, member of the family. For wide channels, the street is close to the Kármán model and the intersection with the origin is transversal, since it was shown to be transversal for the Kármán case. This means that the origin is in the interior of the region (see figure $5 a$ ).

As the channel grows narrower, and $\mu$ increases, the region drifts and, eventually, the intersection with the origin moves to its border. At this point the intersection is no longer transversal and can be expected to disappear for nearby perturbed systems. In fact, for slightly higher values of $\mu$, the region stays tangent to the horizontal axis, but does not cross it in the neighbourhood of the origin. When this happens, there are some segments which never cross into an unstable quadrant, and the vortex street is stable for a finite range of the parameter $\nu$. The three parts of figure 5 show the regions of the $(a, c)$-plane corresponding to the $(\nu, \kappa)$ family for three different values of $\mu$, which are respectively below, above, and at the critical point at which the region leaves the origin. The corresponding stability diagram is shown in figure 6 . 


\section{Discussion and conclusions}

We have shown that the apparently accidental stability properties of some simple models of the Kármán vortex street and, in particular, the presence of isolated, marginally stable, members in one-parameter families of streets, are a consequence of the structure imposed on the space of evolution matrices by the Hamiltonian nature of the equations that govern the flow, and by its symmetries.

This conclusion applies to periodic infinite vortex arrays which have back-to-fore symmetry, and which are close to the point-vortex model for the Kármán street. As such, the stability properties discussed in this paper should apply to a wider set of problems than those discussed here directly and, in particular, they should hold for models of the vortex street that are more general than those tried until now.

Actually, what we have shown is that any family of vortex streets which is close to the point vortex model, and satisfies the proper conditions, has a set of eigenvalues that is close to the eigenvalues of that model, and that this set contains an isolated linearly stable member. In general, any member of such a family might have other eigenvalues that have nothing to do with those of the point-vortex model, and the discussion in this paper says nothing about their properties. In fact, it is perfectly possible that some of those eigenvalues are unstable, making even the vortex street corresponding to the stable member of the original eigenvalue set globally unstable, but the results derived here show that global stability is limited at most to an isolated, marginally stable, member by the behaviour of the original eigenvalues. Actually, in the only case that has been studied numerically in detail, the finite-area model in Meiron et al. (1984), the extra modes introduced by the oscillations of the core shapes remain stable.

Some comment is needed on the meaning of closeness in the above statements and, in general, on the limits of validity of the present results. As discussed in the previous paragraph, stability can be lost (but not gained) from the introduction of new modes of instability by a given model. Obviously, dissipation or loss of back-to-fore symmetry will invalidate the conclusions, but in different ways. Dissipation is completely outside the present discussion, but loss of symmetry will throw us back into the general codimension-3 case (figure 2 ). The marginally stable street will then still be isolated, but we shall need two parameters to find it, instead of one, and the general situation will be more unstable than before.

The question of continuous vorticity distributions is more delicate. As mentioned previously, a Hamiltonian formulation is known for those cases, and the question is whether the collective eigenvalues remain discrete as the model is deformed from point vortices to a continuous vorticity distribution. This is probably so as long as the area of the resulting cores is small with respect to the distance between vortices. The vorticity distribution should mainly influence the eigenvalues associated with the internal oscillations of the cores, which might indeed stop being discrete. But the ratio between these eigenvalues and those of the collective modes is $O\left(L^{2} / \alpha\right)$, were $\alpha$ measures the area of the cores, and, as long as this factor remains large, it is unlikely that both ranges interact strongly enough to spread the discrete collective eigenvalues. In any case, the effect of continuous vorticity distributions should be considered open at the moment.

The most likely cause of failure is loss of transversality, which has been discussed in $\$ 4$, but which should only be expected to occur for models which differ from the point-vortex case by $O(1)$. After transversality is lost the properties of the system depend on the details of the tangency.

The question remains of what is the relation of the present results to natural vortex 
streets. As was mentioned in the Introduction, natural streets do show a degree of instability for sufficiently high Reynolds numbers, even for strictly two-dimensional flows. Saffman \& Schatzman (1982b) have reviewed the matter, and conclude that experimental and theoretical results are at least moderately consistent. Kida (1982) also cites experimental evidence of instability with a wavelength that agrees with his theoretically most unstable value. In all these cases the flow was probably threedimensional (Gerrard 1966), but the present stage of knowledge on strictly twodimensional wakes is not enough to allow a useful comparison with theory.

A different question is the formation of periodicity in real wakes. Actually, the doubly infinite Kármán-street models, and all the theory discussed in this paper, are not applicable to the formation of the vortex street behind a body, since that is clearly not a doubly infinite system, has no back-to-fore symmetry, and is not even in equilibrium, if one takes into consideration the body itself. However, numerical results by Aref \& Siggia (1981), and Kazuhiro \& Oshima (1985), suggest that doubly infinite vortex streets, initially at equilibrium, will rearrange themselves into new stable systems with longer wavelength after an appropriate perturbation, but only if the new system is closer to the critical spacing than the old one. Also, as mentioned earlier, the experimental values for the spacing ratio at which natural streets form seem to be close to the critical one.

Little is known about the nonlinear evolution of unstable vortex streets, but the numerical experiments cited above suggest that they eventually disintegrate into random pairs and triplets and eject closely bound couples of vortices of opposite vorticity which travel at great speeds away from their original positions. This decay mode has actually been observed experimentally in strictly two-dimensional wakes at high Reynolds numbers (Couder et al. 1984), but not at lower ones. All this suggests that the stability of real wakes is indeed a dissipative effect, and that viscosity somehow manages to create a region of stable equilibria near the marginally stable configurations of the inviscid models, which would disintegrate otherwise. These states, although they would obviously decay in the long term owing to dissipation, could actually be attracting in a shorter timescale, and provide the justification for the regular wakes found in natural flows.

While this paper was being circulated as a preprint it came to my attention that similar results had recently been obtained by $R$. S. MacKay. The revised version of the paper owes much to the resulting discussions with him.

This work was accomplished during several visits by the author to the California Institute of Technology, supported in part by a grant from the Department of Energy, Office of Basic Energy Science (DE-AT03-76ER72012). I would like to thank Professor P. G. Saffman for introducing me to the problem and for many illuminating discussions.

\section{Appendix}

The following is a calculation of the codimension of the manifold of matrices with index-1 double real eigenvalues in the space of matrices of form (16). It also gives the behaviour of eigenvalues in the neighbourhood of that manifold. The properties of Hamiltonian matrices that are used here can be found either in Arnol'd (1978) or in Mal'tsev (1963).

It is easy to see from the form of (16) that the right eigenvectors of $\boldsymbol{I} F$ have either the form $\{a, 0,0, b\}$ or $\{0, a, b, 0\}$, and it can be checked by direct calculation that, if $\{a, 0,0, b\}$ is an eigenvector for the eigenvalue $\sigma,\{0,-a, b, 0\}$ is an eigenvector for 
$-\sigma$. Any Hamiltonian matrix can be transformed into block-diagonal form in terms of its projections over real orthogonal invariant subspaces associated with its eigenvalue quartets (or pairs). Consider a full complex quartet that is collapsing into a double real pair. The corresponding diagonal block can be expressed as $\boldsymbol{Y}^{\prime} \mid \boldsymbol{F} X$, where the columns of $X$ are any basis for the associated right-invariant subspace, and those of $\boldsymbol{Y}$ are base vectors for the corresponding left invariant subspace, and such that $\boldsymbol{Y}^{\prime} \boldsymbol{X}=\boldsymbol{U}$.

It is also a general property of Hamiltonian matrices that, if $z$ is a right eigenvector for the eigenvalue $\sigma, I z$ is a left eigenvector for $-\sigma$. Therefore, if $X$ is formed by a quartet of columns

$$
X=\left[\begin{array}{rr}
\boldsymbol{A} & 0 \\
0 & -\boldsymbol{A} \\
0 & \boldsymbol{B} \\
\boldsymbol{B} & 0
\end{array}\right]
$$

where $A$ and $B$ are $(N \times 2), Y$ is formed by the quartet

$$
\left.\begin{array}{l}
\boldsymbol{V}=\mathbf{I X}\left[\begin{array}{cc}
0 & D \\
-D & 0
\end{array}\right], \\
\boldsymbol{D}=\left(\boldsymbol{A}^{\prime} B+B^{\prime} A\right)^{-1}
\end{array}\right\}
$$

The general form of the diagonal block is then

$$
\begin{aligned}
& F_{\sigma}=\left[\begin{array}{cc}
0 & D \\
-D & 0
\end{array}\right] \quad X^{\prime} \| F X=\left[\begin{array}{cc}
D E & 0 \\
0 & -D E
\end{array}\right] \\
& E=\left[A^{\prime}, B^{\prime}\right]\left[\begin{array}{ll}
R_{11} & R_{12} \\
R_{21} & R_{22}
\end{array}\right]\left[\begin{array}{l}
A \\
B
\end{array}\right] .
\end{aligned}
$$

Since both $D$ and $E$ are real and symmetric, and since the choice of the columns of $\boldsymbol{A}$ and $\boldsymbol{B}$ is undetermined up to a linear combination, it is always possible to make one of them diagonal. Therefore, each of the two diagonal sub-blocks in $\boldsymbol{F}_{\sigma}$ is a real $2 \times 2$ matrix which is the product of a diagonal and a symmetric one, and has the form

$$
\left[\begin{array}{cc}
D_{1} E_{11} & D_{1} E_{12} \\
D_{2} E_{12} & D_{2} E_{22}
\end{array}\right] .
$$

These matrices form a four-dimensional space in which the only members with a double eigenvalue of index 1 are multiples of the $2 \times 2$ identity matrix, which are generated by the two conditions

$$
E_{12}=0, \quad D_{1} E_{11}=D_{2} E_{22} \text {. }
$$

Since this set is generated by two restrictions, it has codimension 2.

It is interesting to compute the behaviour of the eigenvalues of (A 4) in the neighbourhood of the index-1 set. In general, they are given by

$$
\sigma=\frac{1}{2}\left(D_{1} E_{11}+D_{2} E_{22}\right) \pm \frac{1}{2}\left[\left(D_{1} E_{11}-D_{2} E_{22}\right)^{2}+4 D_{1} D_{2} E_{12}^{2}\right]^{\frac{1}{2}}
$$

and the set of matrices with double eigenvalues is located at

$$
D_{1} E_{11}-D_{2} E_{22}= \pm 2 E_{12}\left(-D_{1} D_{2}\right)^{\frac{1}{2}} \text {. }
$$

If $D_{1} D_{2}<0$ this set is formed by two codimension-1 real components which intersect at (A 5) and which separate its neighbourhood into four sectors with alternating stability characteristics. This is the situation in figure 3 and in the vortex street. If $D_{1} D_{2}>0,(\mathrm{~A} 7$ ) is only satisfied at (A 5), and there is no exchange of stability when 
the eigenvalue quartet collapses. It is easy to see that the sign of $D_{1} D_{2}$ is maintained under small perturbations if the eigenvalues are bounded away from zero, as is the case in the vortex street, and that, as a consequence, the local structure of the stability map is also maintained under small perturbations. This is a particular case of a general theorem that links the loss of stability of a Hamiltonian system to the signatures of the eigenvalues that coalesce on the collapse of a quartet. A review of these and other related results can be found in (MacKay 1986).

\section{REFERENCES}

AREF, H. \& Siggia, E. D. 1981 Evolution and breakdown of a vortex street in two dimensions. J. Fluid Mech. 109, 435-463.

Arvol'b, V. I. 1969 The Hamiltonian nature of the Euler equations in the dynamics of a rigid body and of an ideal fluid. Usp. Math. Nauk. 24, 225-226.

ARnoL'D, V. I. 1978 Mathematical Methods of Classical Mechanics, p. 230. Springer.

Batchilor, G. K. 1967 An Introduction to Fluid Dynamics, pp. 350-352. Cambridge University Press.

Christiansen, J. P. \& Zabusky, N. J. 1973 Instability, coalescence and fission of finite-area vortex structures. J. Fluid Mech. 61, 219-243.

Couder, Y., Basdevant, C. \& Thomé, H. 1984 Solitary vortex couples in two-dimensional turbulent wakes. C. R. Acad. Sci. Paris II, 299, 89-94.

Dомм, U. 1956 Über die Wirbelstraßen von geringster Instabilität. Z. angew. Math. Mech. 30, 367-371.

GaLis, D. M. 1975 Versal deformation of linear Hamiltonian systems. Trudy Sem. Pet. Vyp. 1, 63-74 (in Russian).

GERRARD, J. H. 1966 The three-dimensional structure of the wake of a circular cylinder. J. Fluid Mech. 25, 143-164.

Kazuhiro, J. H. \& Oshima, Y. 1985 Numerical study of two-dimensional vortex street. In Proc. Intl Symp. Comput. Fluid Dyn., Sept. 1985, Tokyo (ed. K. Oshima), pp. 617-627. Japan Soc. Comp. Fluid Dynamics.

KrDA, S. 1982 Stabilizing effects of finite core in a Kármán vortex street. J. Fluid Mech. 122, 487-504.

LAMB, H. 1945 Hydrodynamics, \$156. Dover.

MacKay, R. S. 1986 Stability of equilibria of Hamiltonian systems. In Nonlinear Phenomena and Chaos (ed. S. Sarkar), pp. 254-270. Bristol: Adam Hilger.

MaL'TSEv, A. I. 1963 Foundations of Linear Algebra, §100-108. Freeman.

Marsden, J. E. \& Weinstein, A. 1983 Coadjoint orbits, vortices and Clebsh variables for incompressible fluids. Physica 7D, 305-323.

Meiron, D. I., Saffman, P. G. \& Schatzman, J. C. 1984 Linear two-dimensional stability of inviscid vortex streets of finite-cored vortices. J. Fluid Mech. 147, 187-212.

Rosenhead, L. 1929 The Kármán street of vortices in a channel of finite breadth. Phil. Trans. R. Soc. Lond. A 208, 275-329.

Safrman, P. G. \& Schatzman, J. C. $1982 a$ Stability of a vortex street of finite vortices. J. Fluid Mech. 117, 171-185.

Saffman, P. G. \& Schatzman, J. C. $1982 b$ An inviscid model for the vortex-street wake. J. Fluid Mech. 122, 467-486.

SHIRER, H. N. \& WELLs, R. 1983 Mathematical Structure of the Singularities at the Transitions Between Steady States in Hydrodynamic Systems. Lectures Notes in Physics, vol. 185, pp. 145-184. Springer.

TANeDA, S. 1959 Downstream development of wakes behind cylinders. J. Phys. Soc. Japan 14, 843-848.

Van Dyke, M. 1982 An Album of Fluid Motion, pp. 56-57. Parabolic. 